\title{
Electrowetting-on-Dielectric System for COVID-19 Testing
}

\author{
Vandana Jain $^{1} \cdot$ K. Muralidhar ${ }^{1}(1)$
}

Received: 14 April 2020 / Revised: 20 May 2020 / Accepted: 23 May 2020 / Published online: 29 May 2020

(C) Indian National Academy of Engineering 2020

\begin{abstract}
The ongoing viral outbreak labeled COVID-19 is spreading rapidly across states and is posing a great threat to public health. Rapid identification of the virus in the population plays a crucial role in isolating the individual and breaking the transmission chain, apart from initiating an appropriate treatment procedure. Here, we discuss an electrowetting-on-dielectric (EWOD) technology that uses a microprocessor-controlled electrode array to merge a possibly infected sample carried by a liquid drop with a drop of a reagent to carry out the testing process. Changes in color occurring during the mixing process of the drops are imaged using a camera.
\end{abstract}

Keywords Digital microfluidics $\cdot$ EWOD $\cdot$ COVID-19 $\cdot$ Droplet merging $\cdot$ Thermal amplification

\section{Introduction}

Electrowetting-on-Dielectric (EWOD) is a device that modifies wettability of a conducting or polarizable liquid drop placed on a dielectric surface using an electrical voltage (Ahmadi et al. 2019; Ugsornrat et al. 2019). In the EWOD device that has been fabricated by the authors, an $8 \times 8$ electrode array (overall dimensions of $40 \mathrm{~mm} \times 40 \mathrm{~mm}$ ) is functionalized to make it hydrophobic with respect to the liquid of interest, mainly water or an aqueous solution. One or more liquid drops are placed on the array. Using a suitable electronic circuit, the electrode array is selectively activated, thus mobilizing the liquid drops towards or away from each other. Both, open and closed configurations have been studied. This arrangement, fabricated in the laboratory, was used originally to study contact line motion, drop deformation and droplet coalescence in the presence of an electric field.

In the PCB-based EWOD device developed on a laboratory scale, basic operations that can be electrically controlled are displacement and mixing, as shown in Fig. 1. These steps are demonstrated from a real experiment with two water droplets, $5 \mu \mathrm{L}$ each in volume. The timescale, namely, the time required for the droplet to cover the distance between adjacent electrodes can be lowered to about $50 \mathrm{~ms}$ at 290

\section{K. Muralidhar}

kmurli@iitk.ac.in

1 Department of Mechanical Engineering, Indian Institute of Technology Kanpur, Kanpur 208016, India
$V_{\mathrm{DC}}$ and increased to a few seconds, as required. Additional timescales, mainly oscillatory, will arise due to liquid inertia, particularly during the merging step, but these coalescence timescales are known to be smaller than $50 \mathrm{~ms}$ for most drop volumes relevant to the device. In EWOD, an asymmetry in the electric field is used to manipulate the droplet motion and merger. Hence, the current drawn by the device is small and the power requirement is in milliwatts $(\mathrm{mW})$. In the experiment shown, the two drops are introduced by a micro-pipette. The circuitry needed to control the external electric field on individual electrode pads and programming of the droplet motion sequence are additional features of the device. For thermal control, the drops are heated from the top side, using an auxiliary electrical circuit. The drop movement is recorded by a camera and analyzed using image processing tools. In Fig. 1, the infected sample is carried in a solution, most likely an aqueous solution and so is the reagent that will chemically react with the sample. In the testing of COVID-19, the infected sample will carry the material from a nasopharyngeal swab and the reagent will be an appropriately chosen enzyme.

Several research groups have developed experimental setups to move liquid drops using an electric field (Ahmadi et al. 2019; Mogi et al. 2019). However, low-cost portable programmable devices for enabling pre-determined motion of one or more drops have only recently been reported (see links given at the end of the article). We are not aware of any such device in the national context. One of the authors reported the preliminary design of such a device in a recent 
Fig. 1 Demonstration experiment on an $8 \times 5$ electrode array showing transport and merging of two water droplets in an EWOD system over a time period of $500 \mathrm{~ms}$

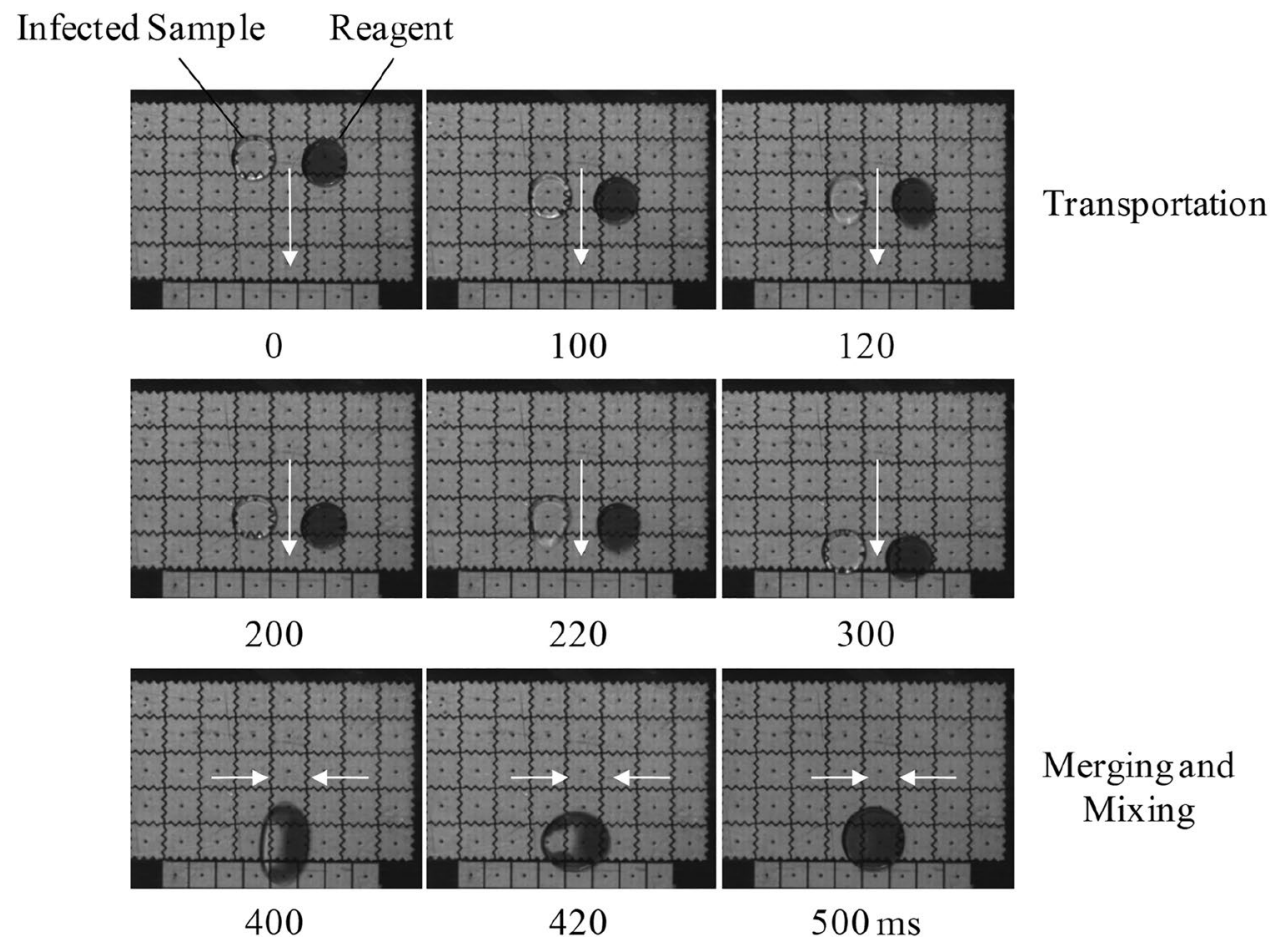

publication (Jain and Patrikar 2020). It has been improved further with multiple capabilities and the present idea builds on these improved features.

\section{Objectives}

We propose to extend the drop manipulation apparatus to testing patient-derived samples for the COVID 19 virus. The method has minimal human intervention and is, hence, safe; it is rapid, and diagnostics rely on a change in color of the liquid drop that follows mixing. Image processing tools are useful here to detect the percentage change of color, in measures such as hue, as well as the appearance of new colors after merger and reaction. In consultation with biomedical researchers and doctors, the process can be tuned in terms of performance and effectiveness.

Polymerase chain reaction (PCR) and isothermal nucleic acid amplification are additional steps needed for the molecular diagnosis of viral infections with a high level of specificity. EWOD provides a new paradigm for PCR application because of its portability, degree of automation, sensitivity, flexibility, reprogrammability, and ease of device fabrication (Coelho et al. 2017). EWOD has found several applications in life sciences including DNA and protein analysis in solutions, since the wetting property on a dielectric of a droplet containing these components is altered by the application of an electrical voltage. When discrete droplets (5-10 $\mu \mathrm{l})$ are placed on an array of independent electrodes, flexibility is improved, waste reduces and the chances of contamination among the health-care workers diminish. Reservoirs carry mixtures of various liquids that are split into multiple droplets, suitable for applications that involve complex, multistep protocols, including RNA extraction and DNA amplification within the EWOD device (Ladha et al. 2020; Fomsgaard and Rosenstierne, 2020). In a similar application, the detection of Dengue II using a PCR-EWOD chip has been demonstrated by Chang et al. (2006). This work shows how the EWOD device and PCR chamber can be integrated on a common substrate. Mixtures of samples derived from a batch of patients can be formed, RNA extracted and tested collectively, particularly with a low-risk population. If the combined sample is free of infection, all participants in the sample can be jointly cleared. This approach can accelerate testing. PCR-EWOD systems available commercially are generic, have multiple features, are sophisticated and expensive, and require expertise in operation. The device proposed here is stand-alone, inexpensive and simpler in terms of ease of fabrication and usage.

\section{Apparatus and Instrumentation}

Following the development of a basic EWOD device, we propose to design and fabricate a portable EWOD-based COVID-19 testing system that has specific advantages of small testing volume, short measurement time, and overall safeguard against contamination. The starting point is a liquid drop to be tested, followed by the introduction of a second reagent drop, to be merged with the first. For a 
positive reaction, the merged drop will show a change in color detectable by a digital camera. In a prototype design (Fig. 2), we will need to incorporate additional arrangements for introducing the drop over the selected electrodes, RNA extraction, and thermal management. RNA extraction and DNA amplification (PCR) can be performed on a shared platform in the EWOD system. Thermal management including cycling is implemented by the surface above using multiple heaters made of ITO glass (Ugsornrat et al. 2010). The speed of device operation can be tuned to the reaction kinetics. The camera will be accompanied by image processing software and IoT functionalities to record and interpret the readings (Paik et al. 2003; Vo et al. 2017). The

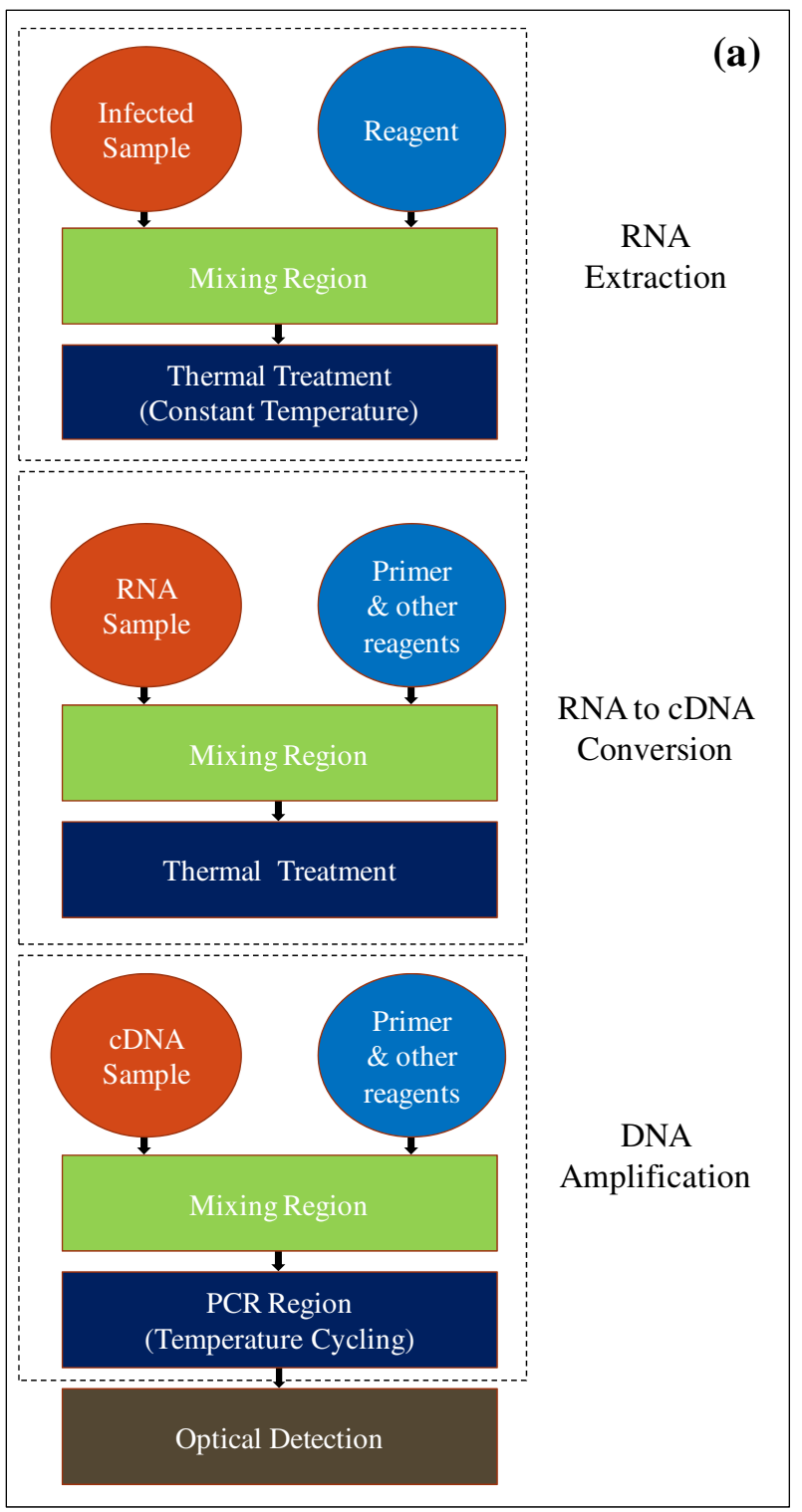

Fig. 2 a Modular layout of an EWOD-based COVID-19 testing system. b Electrode array of the EWOD device divided as per the required functionality device, thus, developed is expected to be of low cost, compact, rapid, and safe.

\section{Discussion}

In the present version of the device, the surface is moderately hydrophobic with an equilibrium contact angle of around $105^{\circ}$. The dielectric layer thickness is of the order of a few microns and the supply voltage needed for droplet movement may be as high as $300 V_{\mathrm{DC}}$. Further improvements in the device are possible along the following lines.

(a) Voltage levels can be brought down to below $50 V_{\mathrm{DC}}$ by increasing the surface hydrophobicity, lowering dielectric constant, and reducing the dielectric layer thickness to below $1 \mu \mathrm{m}$ (Samad and Kouzani 2014).

(b) Pre-treatment of the swab collected from one or more patients in terms of RNA extraction in the EWOD device has been very recently reported but requires implementation in the device proposed.

(c) The choice of the reagent, details of the biochemical reactions, and operating conditions are to be specified. The chemical composition of the reagent that will generate a measurable optical signal on mixing with the sample has to be suitably identified.

(d) Specificity and sensitivity depend on the component specifications of the EWOD system.

(e) Cost A prototype with a performance as shown in Fig. 1 can be assembled at a cost of INR 40,000. Additional devices of RNA extraction and thermal treatment will have to be integrated with it. The measurement ends with a record of the color acquired by the merged drop and consequent interpretation. Thus, a total budget of Rs. 2,00,000/- is anticipated for setting up a stand-alone device in a laboratory.

\section{Closure}

Including RNA extraction, thermal treatment, DNA amplification and a suitably chosen reagent, we propose to convert a droplet actuation EWOD device into an integrated, low-cost, rapid testing device for COVID-19.

\section{Links Related to Droplet Actuation Devices}

These international laboratories report development of EWOD-based droplet actuation apparatus. http://micro fluidics.utoronto.ca/instrumentation.php; http://microfluid ics.ee.duke.edu; https://www.gaudi.ch/GaudiLabs/?page_ id=392; https://tangible.media.mit.edu/project/programmab 


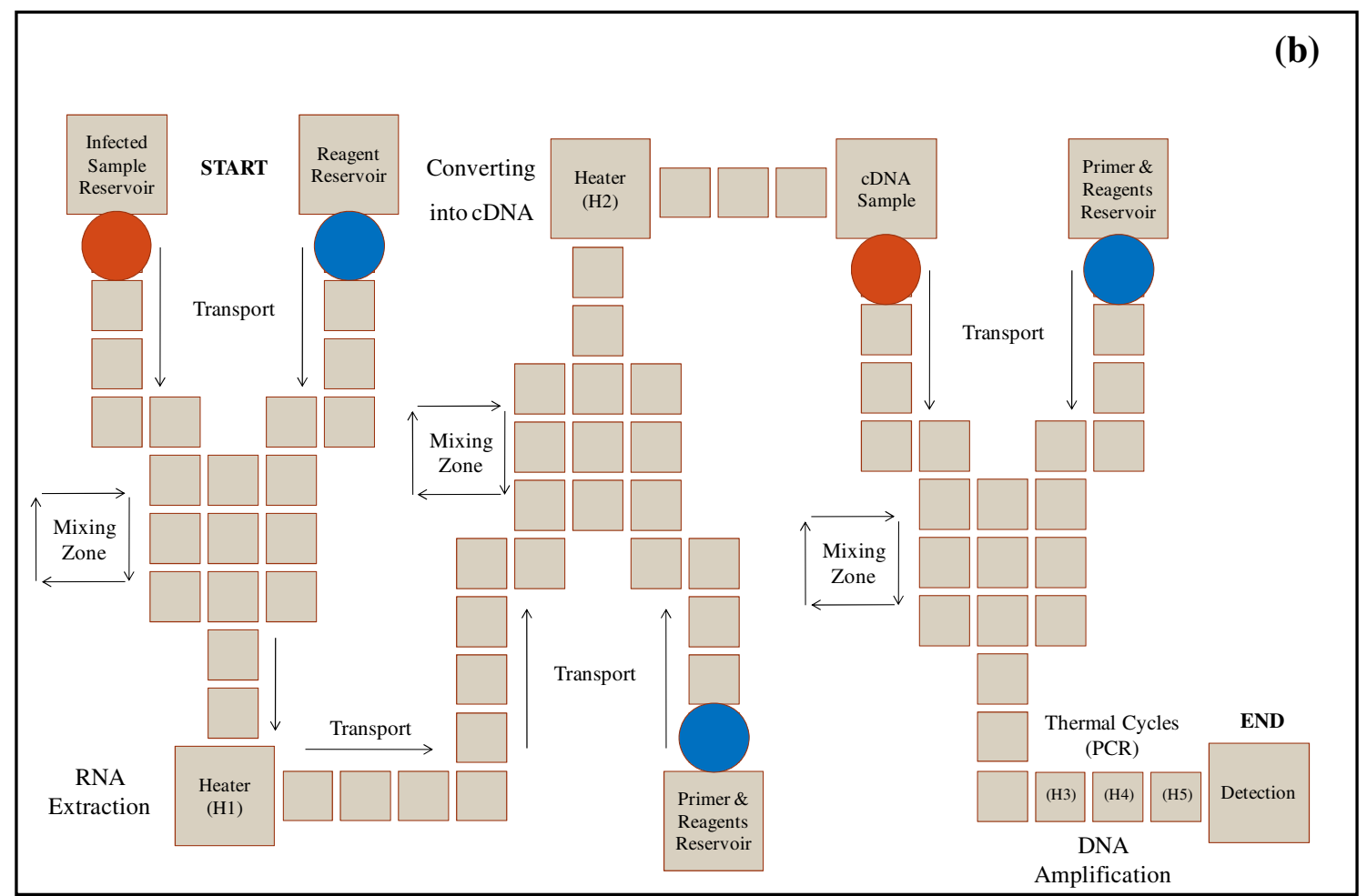

Fig. 2 (continued)

le-droplets/. Survey of these and related links shows that application of EWOD to the testing for COVID-19 remains incomplete.

\section{References}

Ahmadi F, Samlali K, Vo PQN, Shih SCC (2019) An integrated droplet-digital microfluidic system for on-demand droplet creation, mixing, incubation, and sorting. Lab Chip 19:524

Chang Y-H, Lee G-B, Huang F-C, Chen y-Y, Lin J-L (2006) Integrated polymerase chain reaction chips utilizing digital microfluidics. Biomed Microdev 8:215

Coelho B, Veigas B, Fortunato E, Martins R, Águas H, Igreja R, Baptista PV (2017) Digital microfluidics for nucleic acid amplification. Sensor 17:1495

Fomsgaard AS, Rosenstierne MW (2020) An alternative workflow for molecular detection of SARS-CoV-2 - escape from the NA extraction kit-shortage. Copenhagen, Denmark. https://doi. org/10.1101/2020.03.27.20044495 (medRxiv preprint)

Jain V, Patrikar RM (2020) A low-cost portable dynamic droplet sensing system for digital microfluidics applications. IEEE Trans Instrum Meas 69:3623-3630

Ladha A, Joung J, Abudayyeh OO, Gootenberg JS, Zhang F (2020) A 5-min RNA preparation method for COVID-19 detection with RTqPCR protocol. Report of the Howard Hughes Medical Institute, Cambridge MA 02139 USA
Mogi K, Adachi S, Takada N, Inoue T, Natsume T (2019) Electrowetting on Dielectric (EWOD) device with dimple structures for highly accurate droplet manipulation. Appl Sci 9:2406

Paik P, Pamula VK, Pollack MG, Fair RB (2003) Electrowetting-based droplet mixers for microfluidic systems. Lab Chip 3:28-33

Samad MF, Kouzani AZ (2014) Design and analysis of a low actuation voltage electrowetting-on-dielectric microvalve for drug delivery applications. In: 36th Annual International Conference of the IEEE Engineering in Medicine and Biology Society 6: 4423

Ugsornrat K, Afzulpurkar NV, Wisitsoraat A, Tuantranont A (2010) Design, simulation, and experimental study of a droplet-based PCR by EWOD. Sens Mater 22:271-284

Ugsornrat K, Pasakon P, Karuwan C, Sriprachuabwong C, Maturos T, Pogfai T, Wisitsoraat A, Tuantranont A (2019) Low-cost and disposable electrowetting-on-dielectric lab on a chip with an integrated electrochemical detector fabricated by screen-printing process. IEEE Sens J 19:8597

Vo PQN, Husser MC, Ahmadi F, Sinha H, Shih SCC (2017) Imagebased feedback and analysis system for digital microfluidics. Lab Chip 17:3437-3446

Publisher's Note Springer Nature remains neutral with regard to jurisdictional claims in published maps and institutional affiliations. 